\title{
Fungal alternative splicing associates with higher cellular complexity and virulence
}

\author{
K. Grützmann', K. Szafranski², M. Pohl', K. Voigt ${ }^{3}$, A. Petzold ${ }^{2}$, S. Schuster ${ }^{1}$ \\ 'Department of Bioinformatics, Friedrich Schiller University, Jena, Germany \\ ${ }^{2}$ Genome Analysis, Leibniz Institute for Age Research, Fritz Lipmann Institute, Jena, Germany \\ ${ }^{3}$ Leibniz Institute for Natural Product Research and Infection Biology - Hans-Knöll-Institute, Jena, Germany
}

\section{Motivations}

Alternative splicing (AS) is a cellular process that increases a cell's coding capacity from a limited set of genes. Although AS is common in higher plants and animals, its prevalence and abundance in other eukaryotes is mostly unknown. Especially in fungi the involvement of AS in gene expression is of great interest, as many fungal species are human- and plant-pathogenic.

\section{Methods}

We present a genome-wide comparative study of alternative splicing in 28 fungi from the three phyla Ascomycota, Basidiomycota and Mucoromycotina, based on spliced alignments of transcripts. We apply a sophisticated random sampling strategy to accurately estimate pergene AS rates of each species.

Results

We show that our method yields estimates that are independent of available transcript data amounts. Our analysis reveals that a greater fraction of fungal genes than previously expected is alternatively spliced. On average over all fungi with sufficient data, $6.4 \%$ of the genes are affected by AS, with Cryptococcus neoformans and Coccidioidis immitis showing extraordinary rates of $18 \%$ and $13 \%$, respectively. On average, the investigated Basidiomycota show higher rates of AS associated genes (8.6\%) than the Ascomycota (7.2\%, excluding yeasts). We find that fungi with more complex cellular structures and a younger evolutionary age, show higher AS rates and more diverse categories of AS involved genes. Thus, we speculate that AS could facilitate higher celIular complexity in fungi. Furthermore, AS affects genes essential for a pathogenic lifestyle, particularly genes associated with cell rescue and dimorphic switching. We hypothesize that AS is a crucial component of gene regulation and fosters fungal virulence. 